Supporting Information for

\title{
A General Method for Patterning Gradients of Biomolecules on Surfaces Using Microfluidic Networks
}

\author{
Xingyu Jiang ${ }^{1}$, Qiaobing Xu ${ }^{1}$, Stephan K. W. Dertinger ${ }^{1}$, \\ Abraham D. Stroock ${ }^{1}$, Tzung-may Fu ${ }^{2}$ \\ and George M. Whitesides ${ }^{1 *}$ \\ 1. Department of Chemistry and Chemical Biology, \\ 12 Oxford Street, Cambridge, MA 02138 \\ 2. Department of Earth and Planetary Sciences Harvard University, \\ 20 Oxford Street, Cambridge, MA 02138
}

* To whom correspondence should be addressed:

gwhitesides@gmwgroup.harvard.edu 
Supporting information Figure 1. Dimensions of the design of the microfluidic channel that incorporates CAMs.
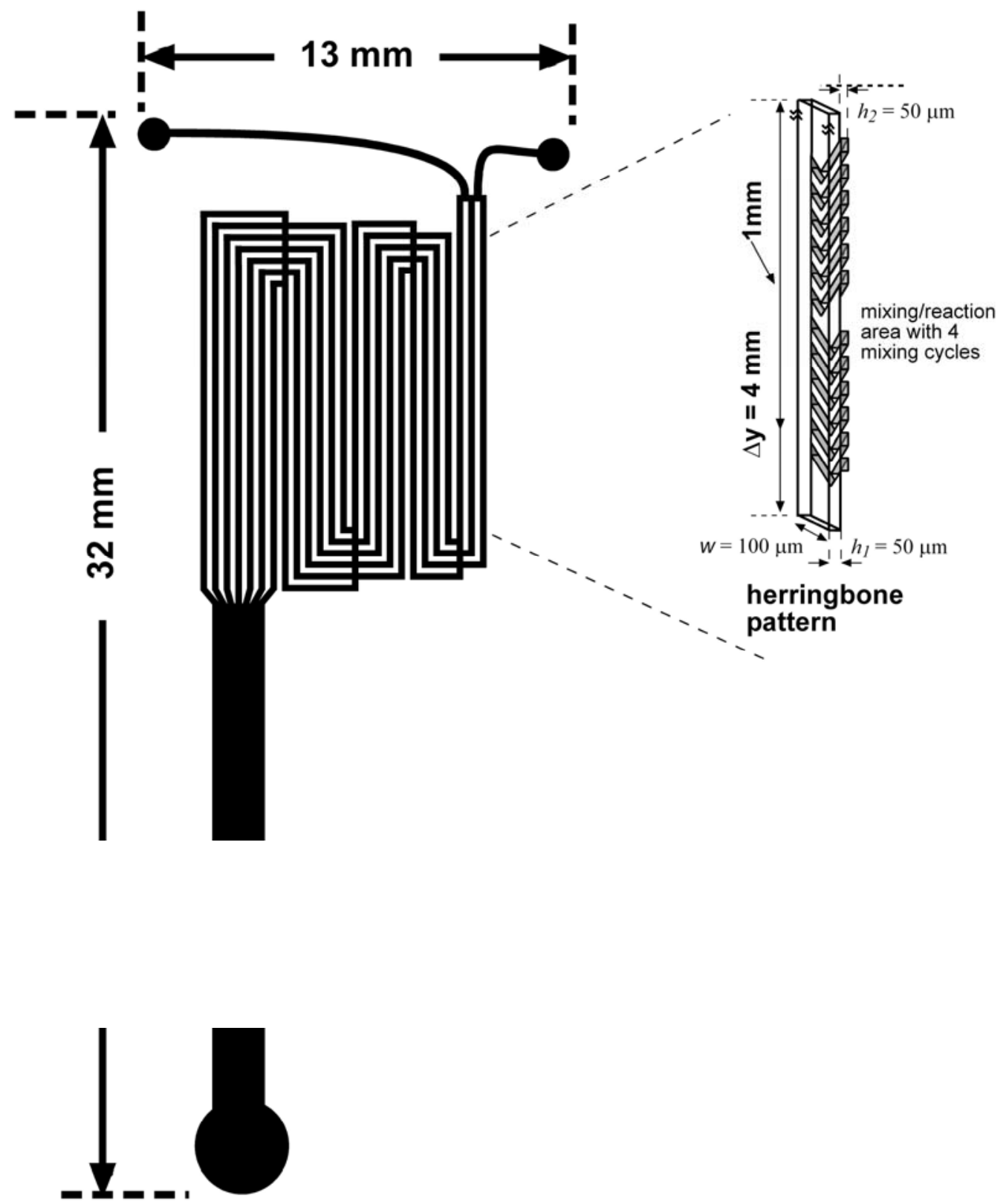